\title{
BEHAVIOUR IDENTIFICATION AND LINEARIZATION of PNEUMatic Rotational Engine
}

\author{
Radek Votrubec, Miroslav Vavroušek
}
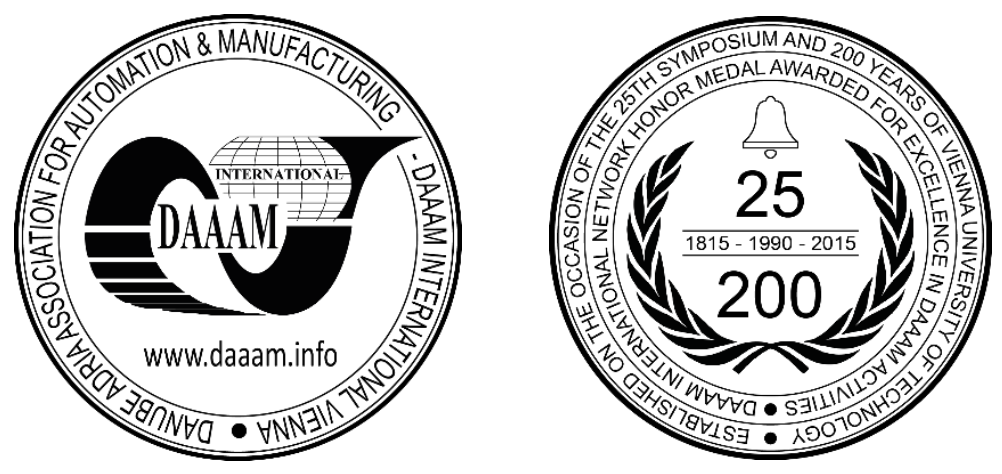

This Publication has to be referred as: Votrubec, R[adek] \& Vavrousek, M[iroslav] (2018). Behaviour Identification and Linearization of Pneumatic Rotational Engine, Proceedings of the 29th DAAAM International Symposium, pp.0287-0293, B. Katalinic (Ed.), Published by DAAAM International, ISBN 978-3-902734-20-4, ISSN 1726-9679, Vienna, Austria

DOI: $10.2507 / 29$ th.daaam.proceedings.041

\begin{abstract}
The article briefly presents behaviour of pneumatic vane rotary motor and approximation of static characteristic of revolutions of pneumatic rotational engine in relation to flow valve control voltage and to working pressure. It deals with basic characteristics of the measured engine. Among measured characteristic there is the influence of the flow and of the working pressure on engine revolutions. Another criterion is stability of rotary movement under different revolution speeds and reaction to the change of the revolution speed. The final approximation will use to linearize control of revolutions of a pneumatic rotational engine. The paper also provide brief information about the progress of creation of approximation function for measured data. The gained information will use for designing the control system of the selected engine.
\end{abstract}

Keywords: pneumatic; linearization; identification; vane rotary motor; static characteristic

\section{Introduction}

Topic of the article are identification measuring of pneumatic motor and approximation of static characteristic for linearization of the control by inversion characteristic. The measured engine is a low performance rotary pneumatic motor. Pneumatic engines are used for many kinds of applications when there is a stable source of compressed air. Among these applications we can name e. g. devices for paint mixing or actuators of assembly lines. The most perspective field of use for this type of propulsion is cargo automotive industry [1], where these motors can be used to run accessories and devices of cargo trucks. The advantage compared to electric motors, which are the most important alternative of today, is high mechanic durableness and high overload capacity of the engine. The disadvantages are notable consumption of the compressed medium and requirements on the distribution system and the source connected with it. As a working medium compressed air is used most often. Compressed air is easily accessible and it is possible to release the compressed air directly into the atmosphere, which lowers the requirements on the quality of the distribution system and the necessity to reverse the airflow in the system. In industrial applications compressor stations are used, which lower the price for unit by mass production. The compressed medium is easily storable in receivers, which can also be used for balancing peak requirements on the compressed medium. 
One of the disadvantages of pneumatic motors can be noisiness, which is caused mostly by releasing the used compressed air into the atmosphere. This problem can be solved by using noise silencers mounted on the pneumatic system mouths. A big advantage of pneumatic engine is also the capability of operating in the areas with explosion risk. Another environment where these engines can be used are the areas with high radioactivity, which damages control circuits of electric motors [2]. Also, compared to electric motors pneumatic engines do not create disturbing electromagnetic field, which can have a negative effect on other devices.

\section{Pneumatic rotary motor}

Rotary pneumatic motors can be divided into four basic groups according to their construction. The first type are gear pneumatic motors. In these engins there is a gearwheel, which seals the space between involute gears. The medium circulates in pockets between the gears and the body and spins the gearwheel. The second important type are piston rotary engines, where the compressed medium moves the pistons. The linear movement of the pistons is transformed to rotary movement. For linear movement transformation to rotary movement a whole set of constructions can be used, e. g. constructions with pistons laid both in the axis of rotation and perpendicularly to the axis. These engines are usually very robust [3]. They can reach high range of revolution speed, but often they are used for lower revolution speed and higher torque. The third type are turbine motors, which are used for very high revolution speed and which comprise of the gas turbine. They are used in hand tools and also in dentist drills. The fourth type are vane rotary motors. These motors are suitable for wide range of revolution speed. The construction solution is based on eccentrically laid rotor in the engine body. In the engine rotor there are canals containing plates. The working space is divided into individual pockets, which are sealed using plates. The plates are made of elastic durable materials. [8] [9]

\section{Measuring structure}

The measuring structure was created for conducting basic engine behaviour tests when the input parameters were changed. The first controlled parameter is working pressure controlled by pressure reduction valve. This valve enables to measure the pressure afterwards using built-in sensor. The second controlled parameter is the airflow controlled by proportional flow valve. The flow valve enables not only to regulate the volume of the flowing air but using function $5 / 3$ switchboard it can also reverse the direction of the air flow, which makes it possible to control the direction of the engine revolutions. The proportional flow valve is connected to the analogue output and is controlled by voltage in the range between 0 and 10 Volts. The controlling elements are supplemented with sensors for measuring main parameters such as the airflow or the revolution speed. The airflow is recorded by flow meter and the revolution speed is measured using incremental rotary counter. The controlling and measuring elements are controlled using the measuring station [4]. The measuring structure consists of two individual parts. One part is dedicated to engine mounting, which enables the structure to be easily adapted for measuring a different engine with different mounting. Both parts are connected by shaft conjunction. The measuring structure is shown in the Fig. 1.

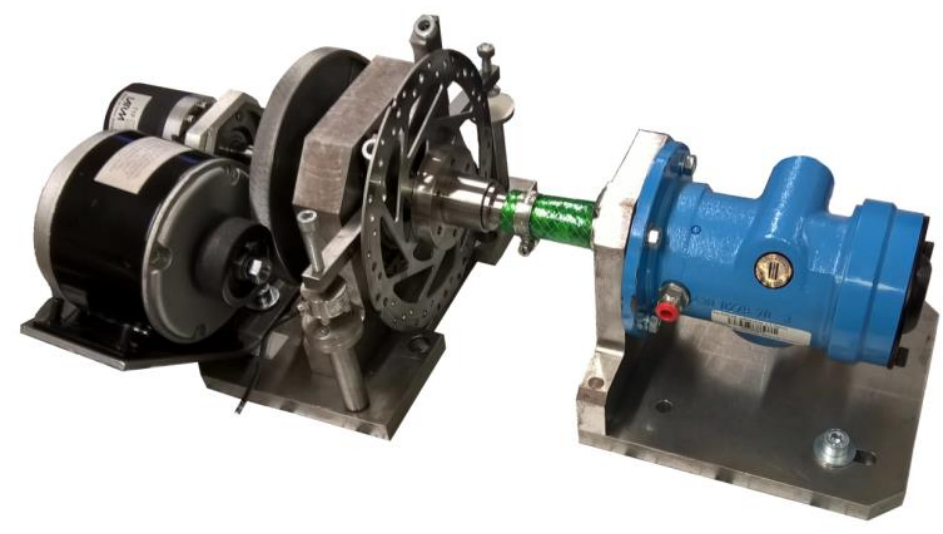

Fig. 1. Measuring structure

Incremental rotary sensor is used to record the position of the shaft. The sensor provides a resolution of 6000 impulses per a revolution and is equipped with a conductor for indication of the zero position of the shaft. The measured signal is transmitted in quadratic coding by the sensor, which enables the data to be easily processed. One of the counters of the measuring station is reserved for the sensor.

The created system is strongly nonlinear in terms of static characteristic. Linearization near the working point did not bring satisfactory results in a wider range of the working revolutions [5]. Approximation of static characteristic was created. Inversion of the resulting approximation was used to linearize the task. A structure for measuring and identifying was used to create a set of input date. The structure was equipped with a revolution sensor and was further used for engine attachment. A scheme capturing the connection of the measuring station to sensors and active elements is shown in the Fig. 2. 


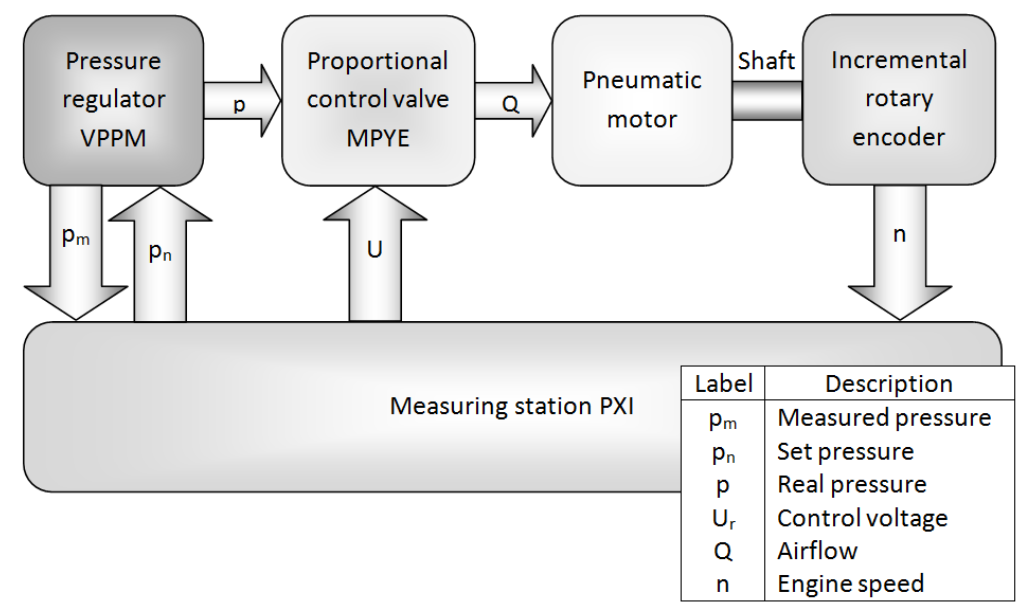

Fig. 2. Scheme of the regulated system

The sensor is an incremental rotary encoder used to measure the shaft turn. The sensor provides a resolution of 6000 impulses per a revolution and is equipped with a conductor for zero position indication. The measuring station is controlled by PC, with which it communicates via Ethernet network. The PC is equipped with a measuring and control software LabVIEW (Laboratory Virtual Instrument Engineering Workbench). The LabVIEW software enables easy creation of virtual measuring instruments [6]. The instrument assembled for identification measurements contains blocks for acquisition of data from the measuring card and blocks for sending the data to output ports. Another parts of the block scheme serve to process the measured data, e.g. to compute the revolutions based on the measured shaft position and time elapsed.

\section{Measuring and analysing data}

For identifying the engine properties on the change of input parameters the dependency of revolution speed on the air flow was measured under various working pressures. There is the dependence of the revolutions on the airflow. The working pressures were measured in the range between $0.1 \mathrm{MPa}$ and $0.4 \mathrm{MPa}$ using $0.05 \mathrm{MPa}$ step. The control voltage of the flow valve was set in the whole range between 0 and $10 \mathrm{~V}$ using a step of $0.1 \mathrm{~V}$. The flow valve was progressively opened in 50 steps. The gained values are captured in the graph in the Fig. 3 . The varying density of the measured points is caused by strong non-linearity of the flow valve. It is evident from the graph that there is nonlinearity caused by increasing loses under higher revolution speeds. Engine run is unstable under low revolutions. This limit was found from the revolution variation and was set to 350 revolutions per minute which roughly corresponds to 5.84 revolution per second. Measured values under this limit were not used in the measured data set. The maximum flow is limited by the distribution system and the working pressure.

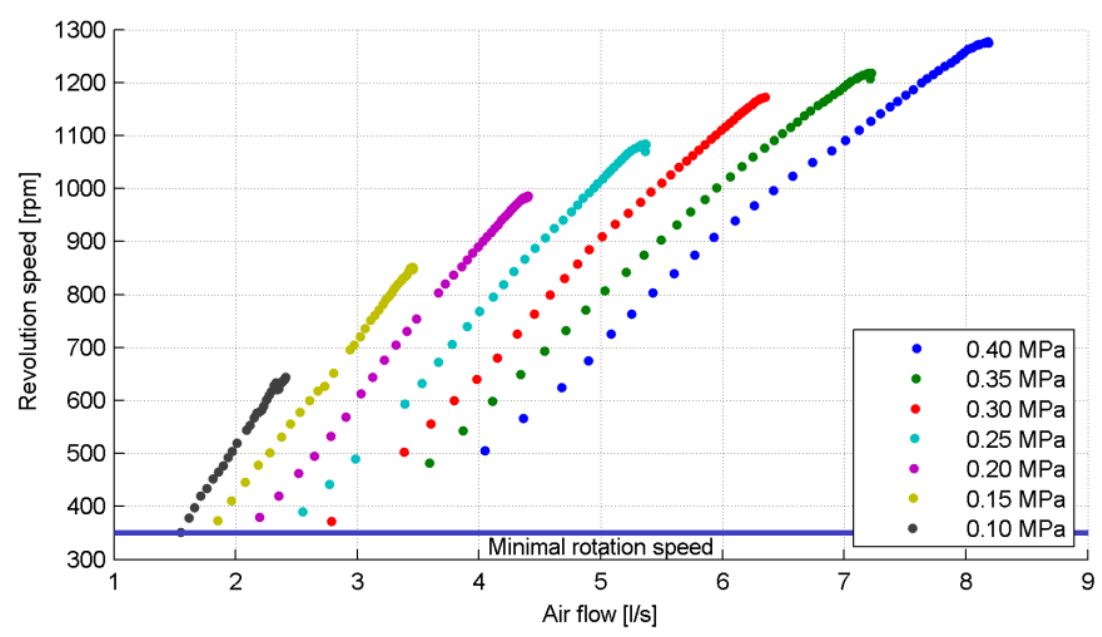

Fig. 3. The dependency of revolution speed on the air flow

To assess the evenness and revolution speed stability of the engine under constant parameters the characteristics of the stable revolution speed was measured. The characteristic of stable revolution speed is captured in Fig. 4. The set of measured values contains 200 samples with interval of $0.01 \mathrm{~s}$ for each step of the proportional flow valve. 


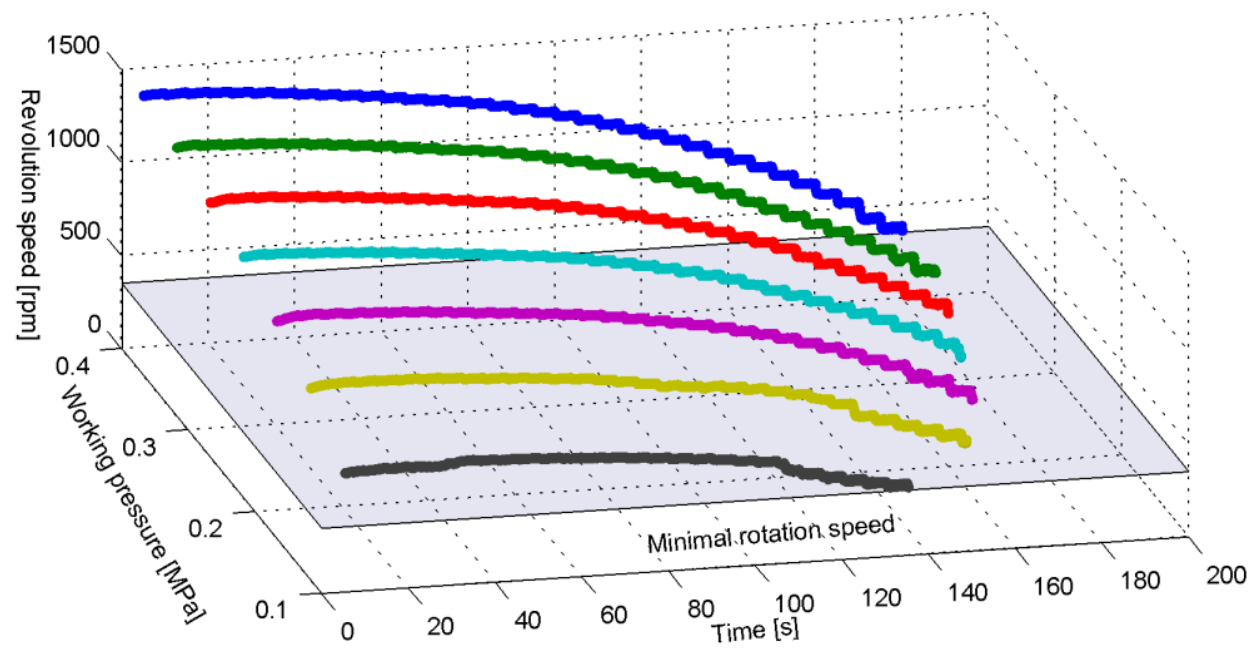

Fig. 4. Stable revolution speed

The proportional flow valve was opened in 50 steps from fully closed to fully open. The measurement was done for various working pressures in the range between $0.1 \mathrm{MPa}$ and $0.4 \mathrm{MPa}$ using step of $0.05 \mathrm{MPa}$. It is evident from the graph that the stability of revolution speed is same with decreasing revolution speed. The engine revolutions for an opening step, which is the 40th step for working pressure of $0.4 \mathrm{MPa}$ is captured in Fig. 5.

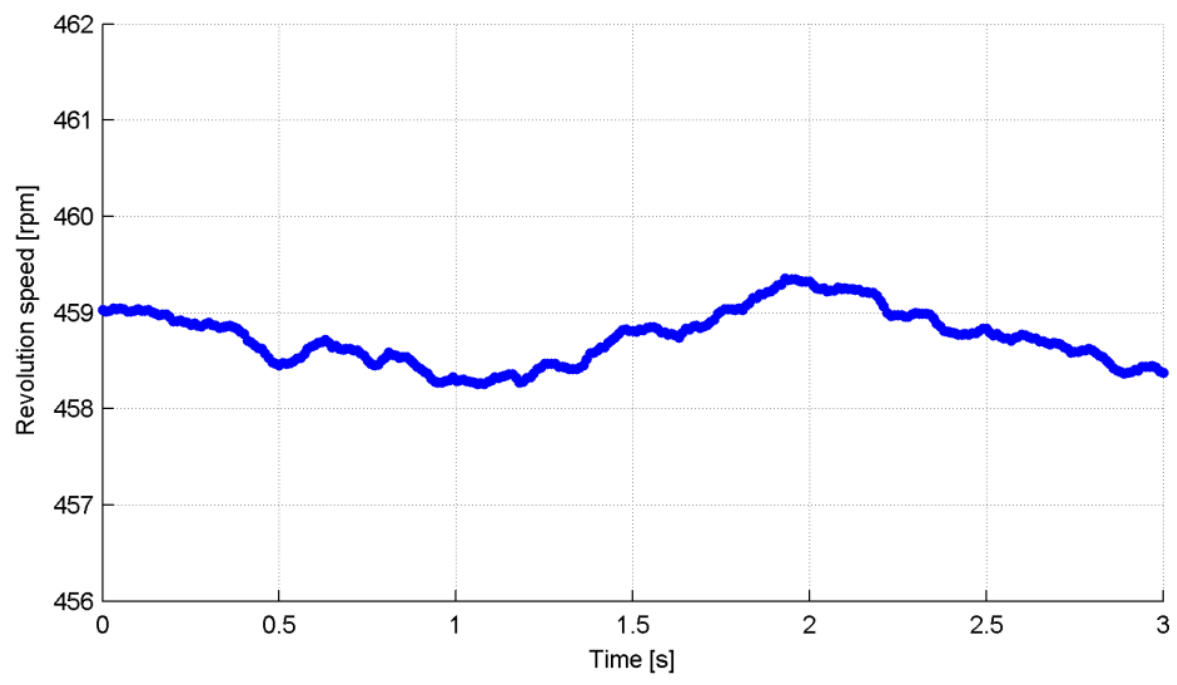

Fig. 5. Stable revolution speed for one step

From the measured values indicators describing revolution speed stability were computed. Table 1 . captures the reduced list for working pressure of $0.4 \mathrm{MPa}$. The first column captures the opening step of the proportional flow valve. Zero step means closed flow valve and step 50 means wholly open flow valve. The second column captures mean value calculated from the measured data. The third column contains the calculated variance of the set and provides information about deviation of the data in the set. The last column describes the maximum deviation.

\begin{tabular}{|c|c|c|c|}
\hline Opening valve step & Average [rpm] & Variance & Maximum deviation \\
\hline 50 & 1351,7 & 0,68 & 1,36 \\
\hline 45 & 1322,2 & 0,36 & 1,23 \\
\hline 40 & 1266,3 & 0,37 & 1,37 \\
\hline 35 & 1189,3 & 0,63 & 1,29 \\
\hline 30 & 1063,2 & 0,48 & 1,67 \\
\hline 25 & 884,1 & 0,19 & 0,95 \\
\hline 20 & 644,4 & 0,29 & 1,54 \\
\hline
\end{tabular}

Table 1. Computed indicators describing revolution speed stability 


\section{Approximation of static characteristic}

In order to approximate the relation of engine revolutions to air flow and working pressure it is necessary to find a function of two variables, whose function value closes to the measured value. The measured values contain a measurement inaccuracy and they are also influenced by processes which unexpectedly interrupt stable engine run [7]. First an approximation of the influence of the working pressure on revolutions is presented. The valve is equipped with a function 5/3, which means that under a control voltage of approximately $5 \mathrm{~V}$ the valve is closed. Control voltage of 10 $\mathrm{V}$ provide a maximum flow and voltage of $0 \mathrm{~V}$ provide it as well, but in the opposite direction. From now on the revolutions in the opposite direction will be shown as negative, which makes it easier to manipulate with the data and to find approximation functions. The graph of measured revolutions clearly shows that the shape of the revolution waveform in relation to control voltage of the flow valve for individual working pressures is still very similar. The waveforms differ in amplification. This amplification increases in direct proportion in relation to pressure. To capture the influence of the pressure on the waveform amplification the revolutions were measured when the flow valve was fully open. The measurement was carried out under pressure ranging from 0.1 to $0.4 \mathrm{MPa}$ using a step of $0.01 \mathrm{MPa}$. The measured waveform of stable revolutions was approximated by a function (1). Function coefficients were found by minimization of the square of deviation of measured and approximated data.

$$
\mathrm{n}_{\max }(\mathrm{p})=24.15-\mathrm{e}^{-0.65 \cdot \mathrm{p}+3.23}
$$

Relation of revolutions when the flow valve is open is captured on the Fig. 6. It is possible to see that the increase in the speed of revolution is smaller under higher pressure, which is caused by the increase in losses related to revolutions. The figure also shows waveform of the approximation function for pressure (1).

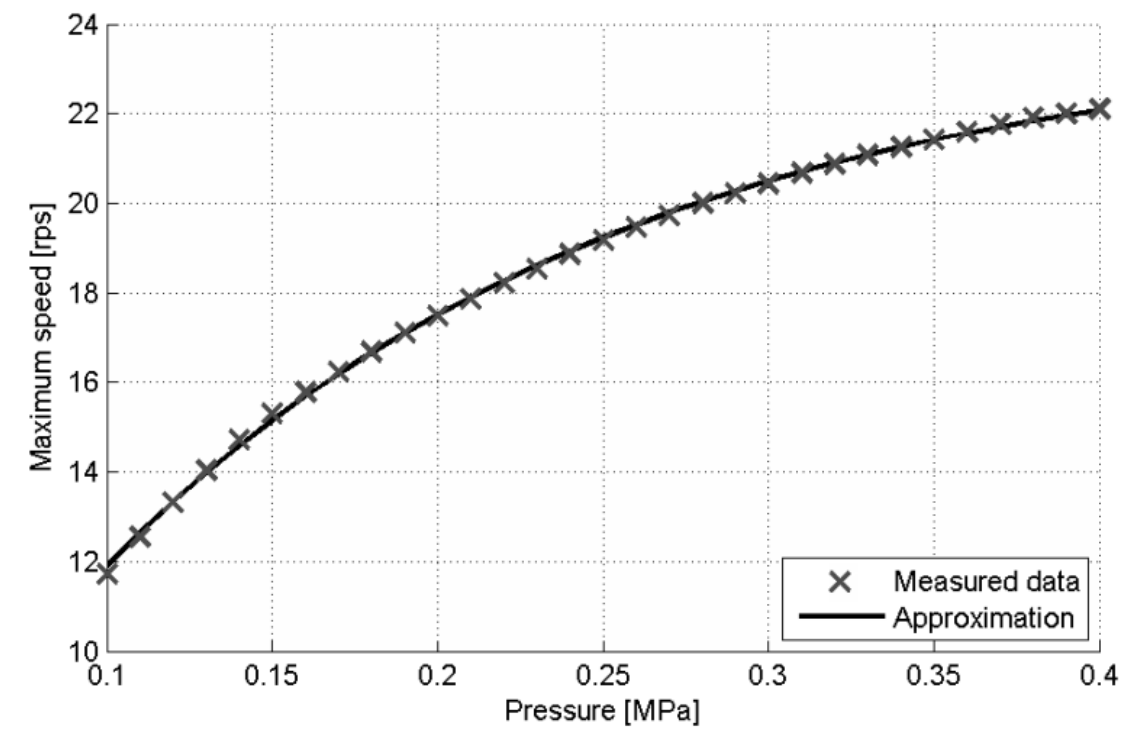

Fig. 6. Relation of revolutions on pressure when valve fully open

Function (1) describing relation of maximum revolutions on pressure will be used to norm waveforms for individual working pressures according to formula (2). This will provide us with normalized revolutions $\mathrm{n}_{\text {norm }}$ in the range between -1 and 1 , where value 1 means maximum revolutions for selected working pressure.

$$
\mathrm{n}_{\text {norm }}(\mathrm{p})=\frac{\mathrm{n}_{\mathrm{mer}}}{\mathrm{n}_{\max }(\mathrm{p})}
$$

Next an approximation of revolution waveform in relation to voltage is described. The waveform shape of the relation of normalized revolutions on control voltage is not possible to approximate by a single function. This property is caused by the hysteresis of the flow valve. Although it is clear from the relation that the waveform is not declining and is therefore possible to divide it into three individual sections. In the first section from 0 to $4 \mathrm{~V}$ the revolutions are in the opposite direction.

$$
\begin{aligned}
& \text { for } U_{n} \leq 4 \quad n_{n o r m}\left(U U_{-} n \leq 4 n\right)=-1.004+e^{-0.7567 \cdot U_{n}+3.448} \\
& \text { for } U_{n}>4 \text { and } U_{n}<6.5 \quad n_{n o r m}\left(U_{n}\right)=0.1873 \cdot U_{n}-1.027
\end{aligned}
$$




$$
\text { for } U_{n} \geq 6.5 \quad n_{\text {norm }}\left(U U_{-} n \leq 4 n\right)=-1.004+\mathrm{e}^{-0.7567 \cdot U_{n}+3.448}
$$

The approximation function of the first section is captured in the formula (3). In the second section from $4 \mathrm{~V}$ to $6.5 \mathrm{~V}$ the motor is instable and the whole are is therefore approximated by a simple linear function. The second section is described by formula (4). In the third section the revolutions are in the normal direction. Approximation function for the third section is captured in the formula (5).

There is an inversion of the approximated functions supplemented by the influence of pressure here. Now there is a function of two independent variables, which will determine the engine revolutions for set control voltage of the flow valve and for the working pressure. Thusly created surface is compared with measured data in the Fig. 7.

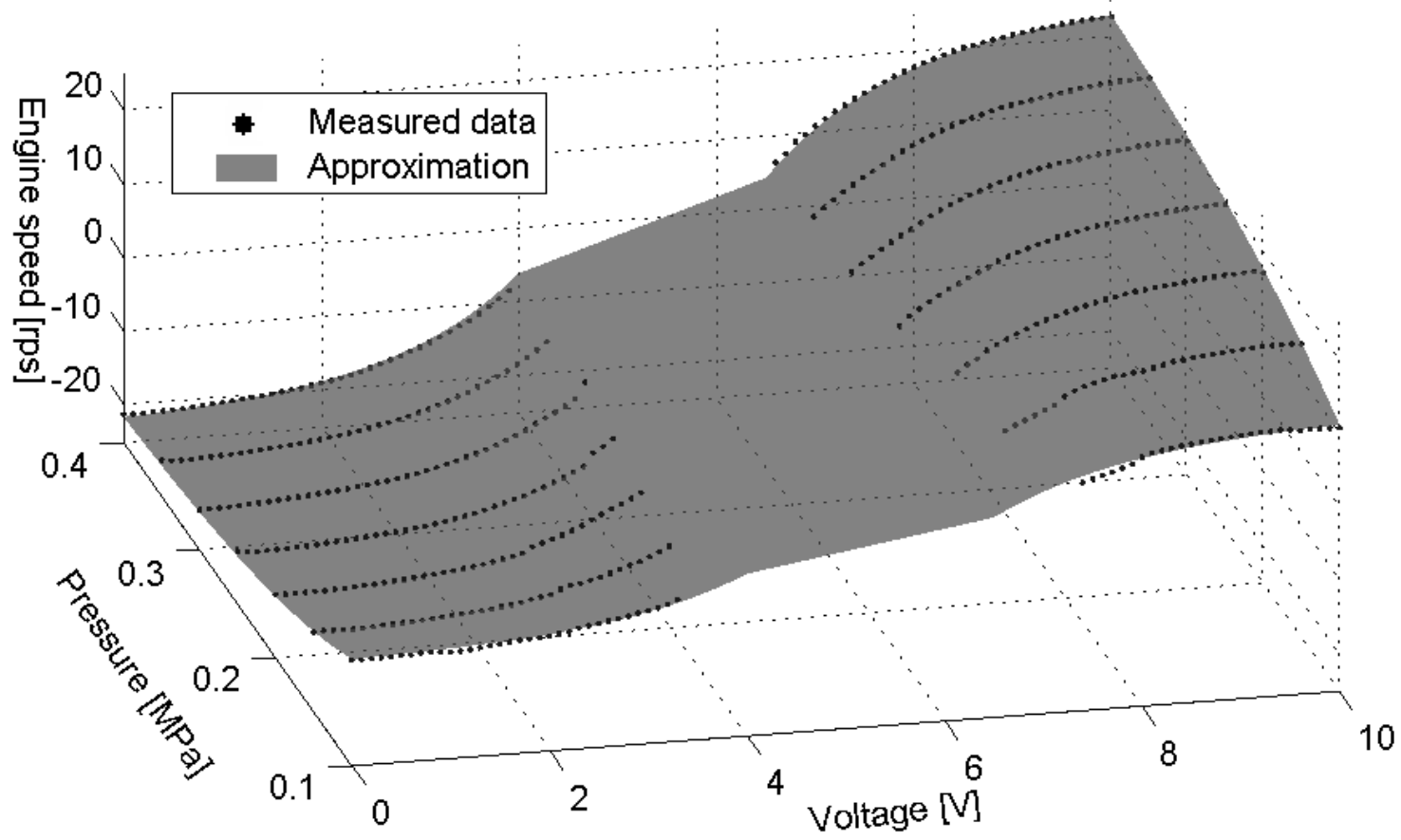

Fig. 7. Measured data and the waveform of the approximation function

The result will be an inverse function, which will determine the value of the control voltage of the flow valve for measured revolutions under selected working pressure. For inverse function the points dividing waveforms of the three sections need to be changed. For the voltage of $4 \mathrm{~V}$ the measured value corresponds to normalized revolutions of -0.278 and for the voltage of $6.5 \mathrm{~V}$ the value corresponds to 0.191 .

These values make the borders of the section of inverse functions. Inverse functions were also supplemented by normalizing the measured revolutions by function value according to selected working pressure from formula (1). Inverse function for the first section is captured in the formula (6). The second section is described by formula (7) and the last section uses formula (8). Compound inverse function was tested during measured data interpretation. The input parameters were working pressure and measured revolutions.

$$
\begin{aligned}
& \text { for } \mathrm{n}_{\max }(\mathrm{p}) \leq-0.278 \quad \mathrm{U}_{\mathrm{v}}(\mathrm{n}, \mathrm{p})=\frac{\ln \left(\frac{\mathrm{n}}{\mathrm{n}_{\max }(\mathrm{p})}+1.004\right)+3.345}{\mathrm{n}_{\max }(\mathrm{p}) \cdot 0.7567} \\
& \text { for } \mathrm{n}_{\max }(\mathrm{p})>-0.278 \text { and } \mathrm{n}_{\max }(\mathrm{p})<0.191 \quad \mathrm{U}_{\mathrm{v}}(\mathrm{n}, \mathrm{p})=\frac{\frac{\mathrm{n}}{\mathrm{n}_{\max }(\mathrm{p})}-1.027}{0.1874} \\
& \text { for } \mathrm{n}_{\max }(\mathrm{p}) \geq 0.191 \quad \mathrm{U}_{\mathrm{v}}(\mathrm{n}, \mathrm{p})=\frac{-\ln \left(1.092-\frac{\mathrm{n}}{\mathrm{n}_{\max }(\mathrm{p})}\right)+4.628}{\mathrm{n}_{\max }(\mathrm{p}) \cdot 0.75282}
\end{aligned}
$$

The calculated voltage was compared with the set control voltage. The ideal state would be an identity. Processed values and the identity are captured in the Fig. 8. From the picture it is clear that the deviations from the ideal state are mainly in the outer regions where there is a small difference between revolutions and voltage. 


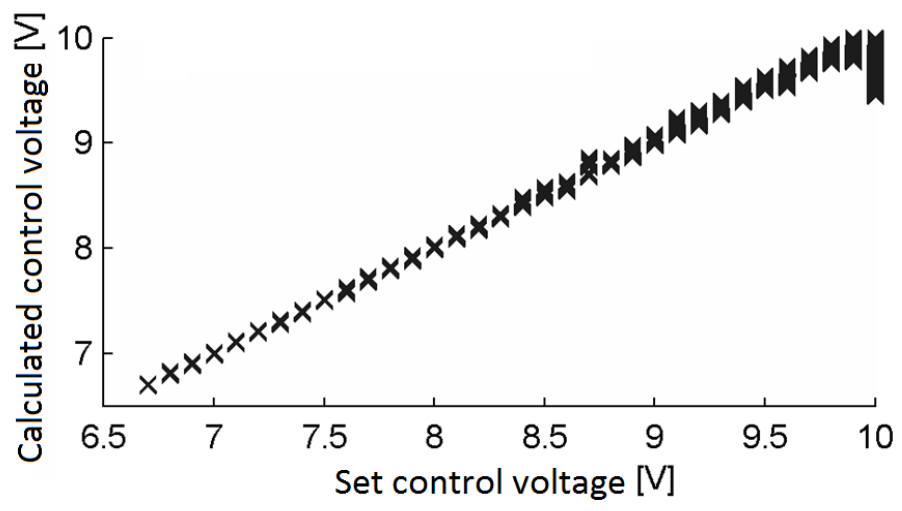

Fig. 8. Comparison of the set and the calculated voltage from measured revolutions

\section{Conclusion}

The article briefly described the identification of the basic properties of pneumatic rotary vane engines. In the article is described identification of rotary pneumatic motor in domain of static characteristic. The article is supplemented by the results of the measurements of basic characteristics focused on the engine revolution stability. According to gathered results the pneumatic rotary engine run is stable enough for most of the applications. The obtained characteristic is approximated at separate intervals using approximation functions. The results obtained will be used in the design of the pneumatic engine control system within the department project. Approximation function and its inverse function was created for the measured data. This approximation will be used for linearization of the task of controlling the pneumatic engine. The reason for designing this approximation function is a strong non-linearity even in a small range of revolutions, which makes it impossible to use classic methods of regulation of linear dynamic systems. A limitation in obtaining measured data is the low accuracy at lower speeds given by construction of motor and low sensitivity of the static characteristic value at higher engine speeds.

\section{Acknowledgments}

The research has been supported by project SGS 21130 "Research and development in the field of 3D technology, manufacturing systems and automation".

\section{References}

[1] Wang, L.; Li, D. F.; Xu, H. X.; Fan, Z. P.; Dou, W. B.; Yu, X. L. (2016). Research on a pneumatic hybrid engine with regenerative braking and compressed-air-assisted cranking, Proceedings of The Institution of Mechanical Engineers Part D-Journal of Automobile Engineering, Vol. 230, No. 3, 406-422, doi: 10.1177/0954407015586706

[2] Tanaka, Y.; Sakama, S.; Nakano, K.; Kosodo, H. (2014). Comparative study on dynamic characteristics of hydraulic, pneumatic, and electric motors, Proceedings of the Asme/Bath Symposium on Fluid Power and Motion Control

[3] Harris, P.; Linke, B.; Spence, S. (2015). An energy analysis of electric and pneumatic ultra-high speed machine tool spindles, 22nd Cirp Conference on Life Cycle Engineering, Vol. 29, 239-244, doi: 10.1016/j.procir.2015.02.046

[4] Bone, G.M.; Xue, M.T.; Flett, J. (2015). Position control of hybrid pneumatic-electric actuators using discrete-

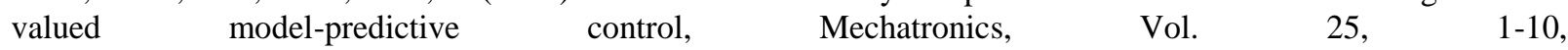
doi: 10.1016/j.mechatronics.2014.10.009

[5] Kosa, P.; Palkova, Z.; Olejar, M. (2015). Model of pneumatic press controlled by PLC, ICERI2015: 8th International Conference of Education, Research and Innovation, 6945-6952

[6] Liu, J. L.; He, F.; Wang, J. H. (2014). Improving pneumatic vane motor's energy efficiency by exhaust air recycling, International Conference on Energy and Power Engineering, 24-29

[7] Lu, C. H.; Hwang, Y. R. (2016). Modeling of an air motor servo system and robust sliding mode controller design, Journal of Mechanical Science and Technology, Vol. 26, No. 4, 1161-1169, doi: 10.1007/s12206-012-0220-2

[8] Hopen J.M.; Hošovský A. (2005), The servo robustification of the industrial robot, Annals of DAAAM for 2005 \& Proceeding 16th International DAAAM Symposium "Intelligent Manufacturing \& Automation: Focus on Young Scientists and Researchers", Vienna, 2005, pp. 161-162.

[9] Židek K.; Šeminský J. (2011), Automated rehabilitation device based on artificial muscles, Annals of DAAAM for 2011 \& Proceeding 22nd International DAAAM Symposium "Intelligent Manufacturing \& Automation: Power of Knowledge and Creativity", Vienna, 2011, pp. 1113-1114. 